\title{
Iris Compression and Recognition using Spherical Geometry Image
}

\author{
Rabab M. Ramadan \\ College of Computers and Information Technology University of Tabuk Tabuk, KSA
}

\begin{abstract}
-this research is considered to be a research to attract attention to the $3 \mathrm{D}$ iris compression to store the database of the iris. Actually, the 3D iris database cannot be found and in trying to solve this problem 2D iris database images are converted to 3D images just to implement the compression techniques used in 3D domain to test it and give an approximation results or to focus on this new direction in research. In this research a fully automated 3D iris compression and recognition system is presented. We use spherical based wavelet coefficients for efficient representation of the 3D iris. The spherical wavelet transformation is used to decompose the iris image into multi-resolution sub images. The representation of features based on spherical wavelet parameterization of the iris image was proposed for the 3D iris compression system. To evaluate the performance of the proposed approach, experiments were performed on the CASIA Iris database. Experimental results show that the spherical wavelet coefficients yield excellent compression capabilities with minimal set of features. Haar wavelet coefficients extracted from the iris image was found to generate good recognition results.
\end{abstract}

Keywords-3D Iris Recognition; Iris Compression; Geometry coding; Spherical Wavelets

\section{INTRODUCTION}

Biometric identification is the process of associating an identity to the input biometric data by comparing it against the enrolled identities in a database [1]. To design and implement robust systems capable of mass deployment, one needs to address key issues, such as human factors, environmental conditions, system interoperability, and image standard [2].The iris, the colored portion of the eye surrounding the pupil, contains unique patterns which are prominent under nearinfrared illumination. These patterns remain stable from a very young age, barring trauma or disease, allowing accurate identification with a very high level of confidence. Commercial iris systems are used as access to secure facilities or other resources, even Criminal/terrorist identification. The enrollment of an individual into a commercial iris system requires capturing one or more images from a video stream [3].

The question arises how to store and handle the acquired sensor data. Typically, the database for such system does not contain actual iris images, but rather it stores a binary file that represents each enrolled iris (the template). Most commercial iris systems today use the Daugman algorithm [4-6]. The recognition system used the template as the input to its process and the iris image is discarded to speed up the recognition process and decrease the storage requirements of the system. From the other point of view, if the data have to be transferred via a network link to the respective location, a minimization of the amount of data must be taken into account. The problem here is that a template alone cannot allow the recreation of the iris image from that it is derived, while the original iris imagery is still valuable for research.

A lot of researches concern on the recognition system which depends on the template of the iris image extracted from the original image. In this paper, the attention is transferred to the iris compression.

This research is considered to be a research to attract attention to the $3 \mathrm{D}$ iris compression to store the database of the iris. Actually, the 3D iris database cannot be found and in trying to solve this problem 2D iris database images are converted to $3 \mathrm{D}$ images just to implement the compression techniques used in 3D domain to test it and give an approximation results or to focus on this new direction in research. These results may encourage researchers to establish a new $3 \mathrm{D}$ iris database image to benefit from all techniques in 3D domain.

Geometry image is an image used to remesh an arbitrary surface onto a completely regular structure [7]. One important use for such a representation is shape compression, the concise encoding of surface geometry [8]. Geometry images can be encoded using traditional image compression and decompression algorithms, such as wavelet-based coders. The mesh-based spherical scheme more natural for coding geometry, and provide good reconstruction of shape detail at very low bit budgets [9].

In this paper, we detail how the geometry image is used to compress the $3 \mathrm{D}$ iris images. The $3 \mathrm{D}$ iris image is mapped to the spherical parameterization domain then the geometry image is obtained as a color image and a surface. Finally, the spherical based wavelet coefficient are computed for efficient representation and compression of the 3D iris image.

The rest of this paper is organized as follows: overview of related work in 3D image compression will be in section II. Section III contains the overview of image preprocessing stage including segmentation and normalization. Spherical geometry image is discussed in section IV. Section V reports the experimental results. Finally, section VI contains the conclusion of this paper.

\section{RELATED WORK}

A major advance in the field of iris recognition results from the expiration of two patents [10]. The first one is the pioneer patent dealing with the general idea of the iris recognition process. It was developed by the ophthalmologists Flom and 
Safir (1987) and it expired in 2005. The second one, developed by the professor John Daugman (1994), was used to protect the iris-code approach and expired in 2011.

Flom and Safir first proposed the concept of automated iris recognition in 1987 [11]. Since then, some researchers worked on iris representation and matching and have achieved great progress [12], [13], [14], [15].

The iris recognition process starts with the segmentation of the iris ring. Further, data is transformed into a double dimensionless polar coordinate system, through the Daugman's Rubber Sheet process. Regarding the feature extraction stage, existing approaches can be roughly divided into three variants: phase-based [16], zero-crossing [17] and textureanalysis methods [18]. Dauman [16] used multi-scale quadrature wavelets to extract texture phase-based information and obtain an iris signature with 2048 binary components. Boles and Boashash [19] calculated a zero-crossing representation of onedimensional (1-D) wavelet transform at various resolution levels of a concentric circle on an iris image to characterize the texture of the iris.. Wildes et al. [20] represented the iris texture with a Laplacian pyramid constructed with four different resolution levels and used the normalized correlation to determine whether the input image and the model image are from the same class. Tisse et al. [21] analyzed the iris characteristics using the analytic image constructed by the original image and its Hilbert transform. Emergent frequency functions for feature extraction were in essence samples of the phase gradient fields of the analytic image's dominant components [22], [23]. Similar to the matching scheme of Daugman, they sampled binary emergent frequency functions to form a feature vector and used Hamming distance for matching. Park et al. [24] used a directional filter bank to decompose an iris image into eight directional subband outputs and extracted the normalized directional energy as features. Iris matching was performed by computing Euclidean distance between the input and the template feature vectors.

Kumar et al. [25] utilized correlation filters to measure the consistency of iris images from the same eye. The correlation filter of each class was designed using the two-dimensional. In [26], Hong and Smith proposed the octave band directional filter banks which are capable of both directional decomposition and an octave band radial decomposition. Finally, in the feature comparison stage, a numeric dissimilarity value is produced, which determines the subject's identity. Here, it is usual to apply different distance metrics (Hamming [16], Euclidian [27] or weighted Euclidian [28]), or methods based on signal correlation [20]. Many image compression and representation methods depend on Gabor analysis or phase information, which are two important components in IrisCode. Daugman demonstrated that Gabor filters are effective for image compression [26]. Behar et al. showed that images can be reconstructed from localized phase [29].

This research is considered to be a research to attract attention to the 3D iris compression to store the database of the iris. Actually, the 3D iris database cannot be found and in trying to solve this problem $2 \mathrm{D}$ iris database images are converted to $3 \mathrm{D}$ images just to implement the compression techniques used in 3D domain to test it and give an approximation results or to focus on this new direction in research. These results may encourage researchers to establish a new 3D iris database image to benefit from all techniques in 3D domain. Geometry images and Spherical representations are used in the compression algorithm.

The construction of a geometry image involves parametrizing a given surface onto a planar domain, and resampling the surface geometry on a regular domain grid. The original work [30] heuristically cuts an arbitrary surface into a disk using a network of cut paths, with $2 \mathrm{~g}$ loops for a genus $\mathrm{g}$ surface. The resulting cut surface is mapped onto a square using a stretch-minimizing parametrization to reduce under sampling.

For shapes with high genus or long extremities, forcing the whole surface to map into a square can introduce high distortion. To mitigate this, we can instead cut the surface into several pieces to produce a multi-chart geometry image [31]. The challenge is to join these piecewise regular charts into a watertight surface.

For genus-zero models, a geometry image may be constructed via spherical parametrization [32], which does not require any a priori surface cuts. The spherical domain is unfolded into a square using a simple cut with elegant boundary symmetries. These boundary symmetries permit the construction of a smooth (C1) polynomial surface, and the regular control grid structure lets the surface be evaluated entirely within the GPU rasterization pipeline [33]. In addition, a spherical geometry image can be compressed using traditional image wavelet Geometry images for static objects can be generalized to geometry videos for animated shapes [34]. Excellent survey of the various 3D mesh compression algorithms has been given by Alliez and C. Gotsman in [34, 30]. The recent development in the wavelet transforms theory has spurred new interest in multi-resolution methods, and has provided a more rigorous mathematical framework. Wavelets give the possibility of computing compact representations of functions or data. Additionally, wavelets are computationally attractive and allow variable degrees of resolution to be achieved. All these features make them appear as an interesting tool to be used for efficient representation of 3D objects.

3D Face recognition is one of the imperative applications calling for compact storage and rapid processing of 3D meshes. Face recognition based on $3 \mathrm{D}$ information is not a new topic. It has been extensively addressed in the related literature since the end of the last century [35-40]. Further surveys of the stateof-the-art in 3D face recognition can be found in [36, 37]. Spherical representations permit to efficiently represent facia surfaces and overcome the limitations of other methods towards occlusions and partial views. In our previous work [41], an innovative fully automated 3D face compression and recognition system is presented. We use spherical based wavelet coefficients for efficient representation of the 3D face. The spherical wavelet transformation is used to decompose the face image into multi-resolution sub images. To the best of our knowledge, the representation of 3D iris point clouds as spherical signals for iris recognition has however not been investigated yet. We therefore propose to take benefit of the 
spherical representations in order to build an effective and automatic 3D iris recognition system.

\section{IRIS IMAGE PREPROCESSING}

Image processing techniques can be employed to extract the unique iris pattern from a digitised image of the eye, and encode it into a biometric template, which can be stored in a database. This biometric template contains an objective mathematical representation of the unique information stored in the iris, and allows comparisons to be made between templates. When a subject wishes to be identified by an iris recognition system, their eye is first photographed, and then a template created for their iris region. This template is then compared with the other templates stored in a database until either a matching template is found and the subject is identified, or no match is found and the subject remains unidentified [42]. There are four main stages of an iris recognition and compression system. They are, image preprocessing, feature extraction and template matching [43], and compression algorithm.

\section{A. Image preprocessing}

The iris image is to be preprocessed to obtain useful iris region. Image preprocessing contains, iris localization that detects the inner and outer boundaries of iris [44], [45] and iris normalization, in this step, iris image is converted from Cartesian coordinates to Polar coordinates. In this paper, We are not focusing on the segmentation instead we are interested in iris compression hence we have used the existing algorithms [42]for image preprocessing normalization feature extraction and segmentation but focusing only on iris compression and matching algorithm. Figure 1 shows the output of the segmentation process using Masek algorithm.
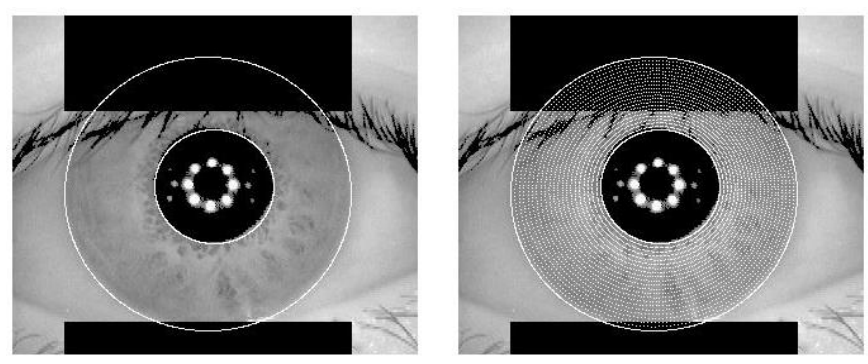

(a)

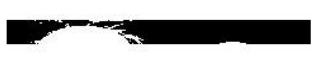

(b)

Fig. 1. Example the output of the segmentation process using Masek algorithm. (a) Automatic segmentation of an iris image from the CASIA database. Black regions denote detected eyelid and eyelash regions. (b) Illustration of the normalization process (polar array - noise array)

\section{B. Feature Extraction}

Feature extraction is the process of getting the iris features, Wavelet transform is used for this purpose.

\section{Template Matching}

Template matching compares the user template with templates from the database using a matching algorithm. The matching metric will give a measure of similarity between two iris templates. Finally, a decision with high confidence level is made through matching methods to identify whether the user is an authentic or imposter.

\section{Compression Algorithm}

Geometry image and spherical wavelet transform will be used for the compression algorithm as shown in the next section. Figure. 2 shows the stages of iris compression algorithm.

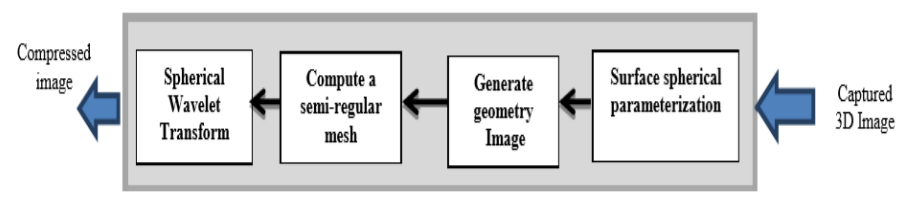

Fig. 2. Stages of iris compression algorithm

\section{SPHERICAL GEOMETRY IMAGE}

Surfaces in computer graphics are commonly represented using irregular meshes. While such meshes can approximate a given shape using few vertices, their irregularity comes at a price, since most mesh operations require random memory accesses through vertex indices and texture coordinates. Also, filter kernels must handle arbitrary mesh neighborhoods, and techniques like morphing, level-of-detail (LOD) control, and compression are complicated. As an alternative, we have introduced the geometry image representation, which captures shape using a completely regular sampling, i.e. a 2D grid of $(\mathrm{x}, \mathrm{y}, \mathrm{z})$ values [46]. The benefits of uniform grids are often taken for granted. Grids allow efficient traversal, random access, convolution, composition, down-sampling, and compression.

\section{A. Spherical Parameterization}

Geometric models are often described by closed, genuszero surfaces, i.e. deformed spheres. For such models, the sphere is the most natural parameterization domain, since it does not require cutting the surface into disk(s). Hence the parameterization process becomes unconstrained [47]. Even though we may subsequently resample the surface signal onto a piecewise continuous domain, these domain boundaries can be determined more conveniently and a posteriori on the sphere. Spherical parameterization proves to be challenging in practice, for two reasons. First, for the algorithm to be robust it must prevent parametric "foldovers" and thus guarantee a 1-to-1 spherical map. Second, while all genus-zero surfaces are in essence sphere-shaped, some can be highly deformed, and creating a parameterization that adequately samples all surface regions is difficult. Once a spherical parameterization is obtained, a number of applications can operate directly on the sphere domain, including shape analysis using spherical harmonics, compression using spherical wavelets [46, 48 ], and mesh morphing [49].

Given a triangle mesh $\mathrm{M}$, the problem of spherical parameterization is to form a continuous invertible map $\varphi: S \rightarrow$ $\mathrm{M}$ from the unit sphere to the mesh. The map is specified by assigning each mesh vertex $\mathrm{v}$ a parameterization $\varphi-1(\mathrm{v}) \in \mathrm{S}$. Each mesh edge is mapped to a great circle arc, and each mesh triangle is mapped to a spherical triangle bounded by these 
arcs. To form a continuous parameterization $\varphi$, we must define the map within each triangle interior. Let the points $\{A, B, C\}$ on the sphere be the parameterization of the vertices of a mesh triangle $\left\{\mathrm{A}^{\prime}=\varphi(\mathrm{A}), \mathrm{B}^{\prime}=\varphi(\mathrm{B}), \mathrm{C}^{\prime}=\varphi(\mathrm{C})\right\}$. Given a point $\mathrm{P}^{\prime}=$ $\alpha \mathrm{A}^{\prime}+\beta \mathrm{B}^{\prime}+\gamma \mathrm{C}^{\prime}$ with barycentric coordinates $\alpha+\beta+\gamma=1$ within the mesh triangle, we must define its parameterization $\mathrm{P}=\varphi-1\left(\mathrm{P}^{\prime}\right)$. Any such mapping must have distortion since the spherical triangle is not developable.

\section{B. Geometry Image}

A simple way to store a mesh is using a compact 2D geometry images. Geometry images was first introduced by $\mathrm{Gu}$ et al. $[46,50]$ where the geometry of a shape is resampled onto a completely regular structure that captures the geometry as a $2 \mathrm{D}$ grid of $[\mathrm{x}, \mathrm{y}, \mathrm{z}]$ values. The process involves heuristically cutting open the mesh along an appropriate set of cut paths. The vertices and edges along the cut paths are represented redundantly along the boundary of this disk. This allows the unfolding of the mesh onto a disk-like surface and then the cut surface is parameterized onto the square. Other surface attributes, such as normals and colors, are stored as additional 2D grids, sharing the same domain as the geometry, with grid samples in implicit correspondence, eliminating the need to store a parameterization. Also, the boundary parameterization makes both geometry and textures seamless. The simple 2D grid structure of geometry images is ideally suited for many processing operations. For instance, they can be rendered by traversing the grids sequentially, without expensive memorygather operations (such as vertex index dereferencing or random-access texture filtering). Geometry images also facilitate compression and level-of-detail control. Figure 3 presents geometric representation of the iris image.

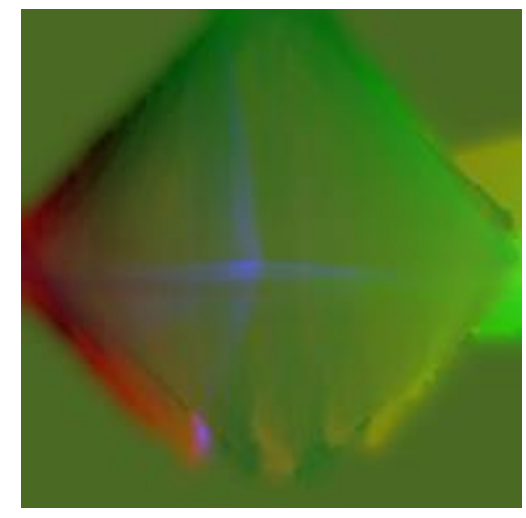

Fig. 3. Geometric representation of the iris image

\section{Wavelet Transform}

- Haar Transform:

Geometry images are regularly sampled 2D images that have three channels, encoding geometric information ( $\mathrm{x}, \mathrm{y}$ and z) components of a vertex in R3 [50]. Each channel of the geometry image is treated as a separate image for the wavelet analysis. The Haar wavelet transform has been proven effective for image analysis and feature extraction. It represents a signal by localizing it in both time and frequency domains. The Haar wavelet transform is applied separately on each channel creating four sub bands LL, LH, HL, and $\mathrm{HH}$ where each sub band has a size equal to $1 / 4$ of the original image. The LL sub band captures the low frequency components in both vertical and horizontal directions of the original image and represents the local averages of the image. Whereas the LH, HL and $\mathrm{HH}$ sub bands capture horizontal, vertical and diagonal edges, respectively. In wavelet decomposition, only the LL sub band is used to recursively produce the next level of decomposition. The biometric signature is computed as the concatenation of the Haar wavelet coefficients that were extracted from the three channels of the geometry image.

\section{- Spherical Wavelets:}

To be able to construct spherical wavelets on an arbitrary mesh, this surface mesh should be represented as a multiresolution mesh, which is obtained by regular 1:4 subdivision of a base mesh [51, 52, 53]. A multi-resolution mesh is created by recursive subdivision of an initial polyhedral mesh so that each triangle is split into four "child" triangles at each new subdivision

Denoting the set of all vertices on the mesh before the jth subdivision as $K(j)$ a set of new vertices $M(j)$ can be obtained by adding vertices at the midpoint of edges and connecting them with geodesics. Therefore, the complete set of vertices at the $\mathrm{j}+1$ th level is given by $K(\mathrm{j}+1)=K(\mathrm{j}) \cup \quad M(\mathrm{j})$. Consequently, the number of vertices at level $\mathrm{j}$ is given by: $10 * 4 j+2$. This process is presented in Figure 4 (a)-(d) where the iris image is shown at 4 different subdivision levels.

In this research, we use the discrete bi-orthogonal spherical wavelets functions defined on a 3-D mesh constructed with the lifting scheme proposed by Schröder and Sweldens [51, 52, 53, 54]. Spherical wavelets belong to second generation wavelets adapted to manifolds with non-regular grids. The main difference with the classical wavelet is that the filter coefficients of second generation wavelets are not the same throughout, but can change locally to reflect the changing nature of the surface and its measure.

They maintain the notion that a basis function can be written as a linear combination of basis functions at a finer, more subdivided level. Spherical wavelet basis is composed of functions defined on the sphere that are localized in space and characteristic scales and therefore match a wide range of signal characteristics, from high frequency edges to slowly varying harmonics [52, 55].

The basis is constructed of scaling functions defined at the coarsest scale and wavelet functions defined at subsequent finer scales. If there exist $\mathrm{N}$ vertices on the mesh, a total of $\mathrm{N}$ basis functions are created, composed of scaling functions and where N0 is the initial number of vertices before the base mesh is subdivided. An interpolating subdivision scheme is used to construct the scaling functions on the standard unit sphere $S$ denoted by $\varphi \mathrm{j}, \mathrm{k}$. The function is defined at level $\mathrm{j}$ and node $\mathrm{k}$ $\epsilon \mathrm{k}(\mathrm{j})$ such that the scaling function at level $\mathrm{j}$ is a linear combination of the scaling function at level $j$ and $j+1$. Index $j$ specifies the scale of the function and $\mathrm{k}$ is a spatial index that specifies where on the surface the function is centered. Using these scaling functions, the wavelet $\psi_{-}(\mathrm{j}, \mathrm{m})$ at level $\mathrm{j}$ and node $\mathrm{m} \in \mathrm{M}(\mathrm{j})$ can be constructed by the lifting scheme. 

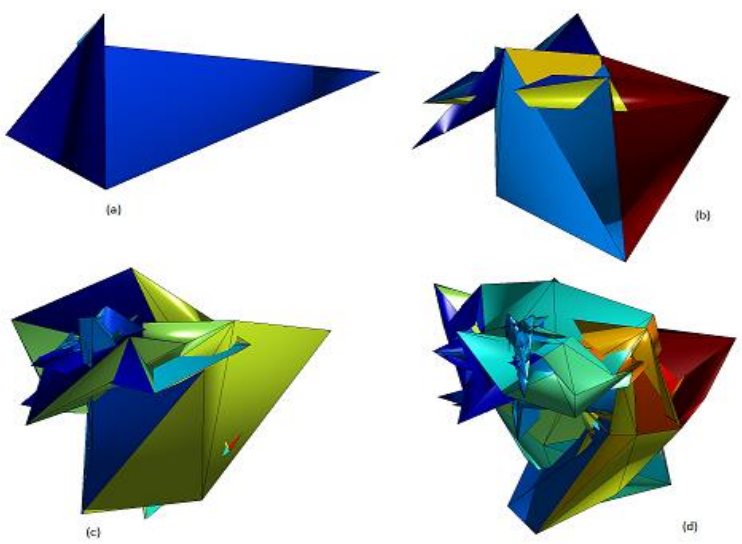

Fig. 4. Visualization of recursive partitioning of the iris mesh at different subdivision levels. (a) Initial icosahedron (scale 0). (b) Single partitioning of icosahedron (scale 1). (c) Two recursive partitioning of icosahedron (scale 2). (d) Three recursive partitioning of icosahedron (scale 3)

A usual shape for the scaling function is a hat function defined to be one at its center and to decay linearly to zero. As the $\mathrm{j}$ scale increases, the support of the scaling function decreases. A wavelet function is denoted by $\psi_{-}(\mathrm{j}, \mathrm{k}): \mathrm{S} \rightarrow \mathrm{R}$. The support of the functions becomes smaller as the scale increases. Together, the coarsest level scaling function and all wavelet scaling functions construct a basis for the function space L2:

$$
L^{2}=\left\{\varphi_{0, k} \mid k \in N_{o}\right\} \cup\left\{\psi_{j, m} \mid j \geq 0, m \in N_{j+1}\right\}
$$

A given function $\mathrm{f}: \mathrm{S} \rightarrow \mathrm{R}$ can be expressed in the basis as a linear combination of the basis functions and coefficients

$$
f(x)=\sum_{k} \lambda_{0, k} \varphi_{0, k}(x)+\sum_{0 \leq j} \sum_{m} \gamma_{j, m} \psi_{j, m}(x)
$$

Scaling coefficients $\lambda_{-}(0, \mathrm{k})$ represent the low pass content of the signal $\mathrm{f}$, localized where the associated scaling function has support; whereas, wavelet coefficients $\gamma_{-}(\mathrm{j}, \mathrm{m})$ represent localized band pass content of the signal, where the band pass frequency depends on the scale of the associated wavelet function and the localization depends on the support of the function. Figure $5(\mathrm{a})-(\mathrm{g})$ presents the spherical wavelets of the iris image.

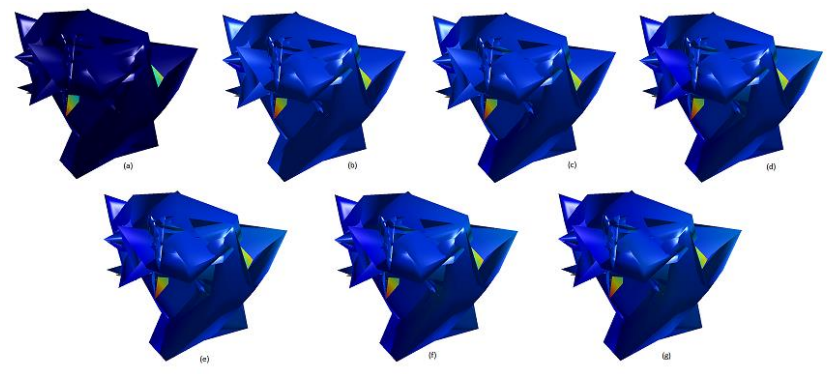

Fig. 5. Spherical wavelet transform of iris image. (a) using 5\% of wavelet coefficients (b) using $10 \%$ of wavelet coefficients (c) using $20 \%$ of wavelet coefficients (d) using $40 \%$ of wavelet coefficients (e) using $60 \%$ of wavelet coefficients (f) using $80 \%$ of wavelet coefficients (g) Using all coefficients

\section{EXPERIMENTAL RESULTS}

The CASIA-IrisV4 data base was used to evaluate the performance of the proposed system. CASIA- IrisV4 is said to be an extension of CASIA-IrisV3 and contains six subsets. It contains a total of 54,601 iris image from more than 1,800 genuine subject and 1,000 virtual subjects. All iris images are 8 bit gray-level JPEG files. In our experiment CASIA-IrisInterval and CASIA-Iris-Lamp will be used.

\section{A. Iris Recognition}

In this experiment, CASIA-Iris-Interval contains 249 subjects. Only 99 subjects will be included in this experiment. For each one only 7 images for each eye is taken. The total number of classes is 198 which have 1386 images. 2dimentional Haar wavelet transform is applied to the templates of the iris images which have dimension of $(20 * 480)$ for each image. Each application of the Haar wavelet decomposition reduces the size of the image to $1 / 4$ of its original size so the input to the $\mathrm{K}$-fold cross validation method is a pattern of $(10 *$ 240) features.

$\mathrm{K}$-fold cross-validation is a statistical method used to evaluate the performance of a learning algorithm [9]. In K-fold cross validation, the input data is divided into $\mathrm{k}$ nearly equal subsets. $\mathrm{K}$ iterations are performed. In each iteration, one of the $\mathrm{k}$ subsets is considered the test set while the other $\mathrm{k}-1$ subsets are put together to form a training set. The output is the average error across all $\mathrm{k}$ trials.

The average recognition rate is $94 \%$ is achieved in this experiment. This result confirms that iris recognition is a reliable and accurate biometric technology. But as mentioned before, the iris recognition is not the objective of this research. We want to focus on 3D iris compression image which will be discussed in the next experiments.

\section{B. Iris compression}

In this experiment, the algorithm is applied to two sets from CASIA Iris-V4, CASIA-Iris-Interval and CASIA-Iris-Lamp. The input image is converted to $3 \mathrm{D}$ image then generate the geometry image. After this, a semi-regular mesh from a gim file is computed. Finally, the spherical wavelet transform on the mesh is computed. The total number of the features after computing wavelet transform is 774 features by keeping only the biggest coefficients. Different percentages of these coefficients were tested and each time the inverse wavelet transform was used to reconstruct the iris image. Figure 6 (a)(g) shows the reconstructed images for the iris using different percentages of wavelet coefficient. These figure shows us that there is no visually difference between the original image and the corresponding reconstructed images.

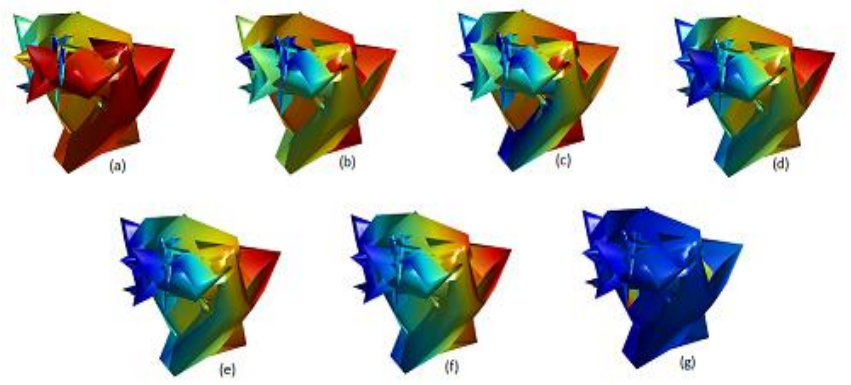

Fig. 6. Wavelet approximation of iris image. (a) using $2 \%$ of wavelet coefficients (b) using $5 \%$ of wavelet coefficients(c) using $10 \%$ of wavelet coefficients (d) using $20 \%$ of wavelet coefficients (e) Using all coefficient 
To evaluate the quality of the reconstructed iris image, the Normalized Error (NE) and Normalized Correlation (NC) were used to. NE is given as follows:

$$
N E=\frac{\|x-y\|}{\|x\|}
$$

Where $\mathrm{X}$ is the original image and $\mathrm{Y}$ is the reconstructed image. i.e. $\mathrm{NE}$ is the norm of the difference between the original and reconstructed signals, divided by the norm of the original signal. The NC is given:

$$
N C=\frac{\sum_{i=1}^{M} \sum_{j=1}^{N} X(i, j) Y(i, j)}{\sum_{i=1}^{M} \sum_{j=1}^{N} X(i, j) X(i, j)}
$$

Where $\mathrm{MxN}$ is the size of the image. The NE and the NC values of the reconstructed images are presented in Table () for the different wavelet subsets.

TABLE I. NE AND NC FOR VARIOUS WAVELET SUBSETS FOR IRIS IMAGE FROM CASIA-IRIS-INTERVAL SUBSET

\begin{tabular}{|l|l|l|l|l|l|l|l|}
\hline Percentage & $\mathbf{5 \%}$ & $\mathbf{1 0 \%}$ & $\mathbf{2 0 \%}$ & $\mathbf{4 0 \%}$ & $\mathbf{6 0 \%}$ & $\mathbf{8 0 \%}$ & $\mathbf{1 0 0 \%}$ \\
\hline NE & 0.477 & 0.1662 & 0.0912 & 0.0664 & 0.0517 & 0.0446 & 0.0311 \\
\hline Nc & 0.6165 & 0.9278 & 0.9614 & 0.9671 & 0.977 & 0.9795 & 0.9979 \\
\hline $\begin{array}{l}\text { No. of } \\
\text { coefficient }\end{array}$ & 39 & 77 & 155 & 310 & 464 & 619 & 774 \\
\hline
\end{tabular}

TABLE II. NE AND NC FOR VARIOUS WAVELET SUBSETS FOR IRIS IMAGE FROM CASIA-IRIS-LAMP SUBSET

\begin{tabular}{|l|l|l|l|l|l|l|l|}
\hline Percentage & $\mathbf{5 \%}$ & $\mathbf{1 0 \%}$ & $\mathbf{2 0 \%}$ & $\mathbf{4 0 \%}$ & $\mathbf{6 0 \%}$ & $\mathbf{8 0 \%}$ & $\mathbf{1 0 0 \%}$ \\
\hline NE & 0.3593 & 0.0411 & 0.0275 & 0.0210 & 0.0166 & 0.0130 & 0.0107 \\
\hline Nc & 0.7318 & 0.9757 & 0.9959 & 1 & 0.9980 & 0.9973 & 0.9993 \\
\hline $\begin{array}{l}\text { No. of } \\
\text { coefficient }\end{array}$ & 39 & 77 & 155 & 310 & 464 & 619 & 774 \\
\hline
\end{tabular}

The NE and NC values indicate that the reconstructed images are the very similar to the original image. In the case of using only $20 \%$ of the wavelets coefficients, the relative error of reconstruction is $9 \%$. The reconstructed signal retains approximately $96.14 \%$ of the energy of the original signal while the number of coefficient is only 155 .

\section{CONCLUSION}

In this paper an innovative approach for 3D iris compression and recognition based on spherical wavelet parameterization was proposed. First. The iris image is to be preprocessed to obtain useful iris region. Image preprocessing contains, iris localization that detects the inner and outer boundaries of iris and iris normalization, in this step, iris image is converted from Cartesian coordinates to Polar coordinates. We are not focusing on the segmentation instead we are interested in iris compression hence we have used the existing algorithms for Masek. Next, the spherical wavelet features were extracted which provide a compact descriptive biometric signature. Spherical representation of iris permits effective dimensionality reduction through simultaneous approximations. The dimensionality reduction step preserves the geometry information, which leads to high performance matching in the reduced space. Multiple representation features based on spherical wavelet parameterization of the iris image were proposed for the 3D iris compression and recognition. The database CASIA was utilized to test the proposed system. Experimental results show that the spherical wavelet coefficients yield excellent compression capabilities with minimal set of features. Furthermore, it was found that Haar wavelet coefficients extracted from the templet of the iris yield good recognition results.

\section{ACKNOWLEDGEMENTS}

The authors would like to acknowledge financial support for this work from the Deanship of Scientific Research (DSR), University of Tabuk, Tabuk, Saudi Arabia, under grant no. 0024/1435.

\section{REFERENCES}

[1] A. Jain, A. Ross, and S. Prabhakar, "An Introduction to Biometric Recognition," IEEE Transactions on Circuits and Systems for Video Technology, vol. 14, no. 1, pp. 4-20, January 2004.

[2] Rakshit, Soumyadip; Monro, Donald M., "An Evaluation of Image Sampling and Compression for Human Iris Recognition," Information Forensics and Security, IEEE Transactions on , vol.2, no.3, pp.605,612, Sept. 2007 doi: 10.1109/TIFS.2007.902401

[3] Ives, R.W.; Bishop, D.A.D.; Yingzi Du; Belcher, C., "Effects of image compression on iris recognition performance and image quality," Computational Intelligence in Biometrics: Theory, Algorithms, and Applications, 2009. CIB 2009. IEEE Workshop on, vol., no., pp.16,21, March 30 2009-April 22009

[4] J. Daugman "How iris recognition works," IEEE Trans. on Circuits and Systems for Video Technology., Vol. 14, No. 1, pp. 21-30.

[5] J. G. Daugman, "High confidence visual recognition of persons by a test of statistical independence," IEEE Trans. Pattern Analysis and Machine. Intelligence, vol. 15, no. 11, pp. 1148 1161, Nov. 1993.

[6] J. G. Daugman, "The importance of being random: Statistical principles of iris recognition," Pattern Recognition, vol. 36, no. 2, pp. 279-291, Feb. 2003.

[7] Gu, Xianfeng, Steven J. Gortler, and Hugues Hoppe. "Geometry images." ACM Transactions on Graphics (TOG) 21.3 (2002): 355-361.

[8] Hoppe, Hugues, and Emil Praun. "Shape compression using spherical geometry images." Advances in Multiresolution for Geometric Modelling. Springer Berlin Heidelberg, 2005. 27-46.

[9] Blum, Avrim, Adam Kalai, and John Langford. "Beating the hold-out: Bounds for k-fold and progressive cross-validation." Proceedings of the twelfth annual conference on Computational learning theory. ACM, 1999.

[10] J. Daugman, "Statistical richness of visual phase information: Update on recognizing persons by iris patterns," Int. J. Comput. Vis., vol. 45, pp. $25-38,2001$

[11] L. Flom and A. Safir, "Iris Recognition system," U.S. Patent 4641 394, 1987.

[12] R. Johnson, "Can iris patterns be used to identify people?," Chemical and Laser Sciences Division LA-12 331-PR, Los Alamos Nat. Lab., Los Alamos, CA, 1991.

[13] K. Bae, S. Noh, and J. Kim, "Iris feature extraction using independent component analysis," in Proc. 4th Int. Conf. Audio- and Video-Based Biometric Person Authentication, 2003, pp. 838-844.

[14] J. Daugman, "Biometric personal identification system based on iris analysis," U.S. Patent 5291 560, 1994.

[15] R.Wildes, J. Asmuth, S. Hsu, R. Kolczynski, J. Matey, and S. Mcbride, "Automated, noninvasive iris recognition system and method," U.S. Patent 5 572 596, 1996.

[16] J. G. Daugman, "Phenotypic versus genotypic approaches to face recognition," in Face Recognition: From Theory to Applications. Heidelberg: Springer-Verlag, 1998, pp. 108-123.

[17] W. Boles and B. Boashash, "A human identification technique using images of the iris and wavelet transform," Signal Processing, IEEE Transactions on, vol. 46, no. 4, pp. 1185-1188, April 1998. 
[18] R. P. Wildes, "Iris recognition: an emerging biometric technology," Proceedings of the IEEE, vol. 85, no. 9, pp. 1348-1363, September 1997.

[19] W. Boles and B. Boashash, "A human identification technique using images of the iris and wavelet transform," IEEE Trans. Signal Processing, vol. 46, pp. 1185-1188, Apr. 1998.

[20] R. Wildes, J. Asmuth, G. Green, S. Hsu, R. Kolczynski, J. Matey, and S. McBride, "A machine-vision system for iris recognition," Mach. Vis. Applic., vol. 9, pp. 1-8, 1996.

[21] C. Tisse, L. Martin, L. Torres, and M. Robert, "Person identification technique using human iris recognition," in Proc. Vision Interface, 2002, pp. 294-299.

[22] T. Tangsukson and J. Havlicek, "AM-FM image segmentation," in Proc. EEE Int. Conf. Image Processing, 2000, pp. 104-107.

[23] J. Havlicek, D. Harding, and A. Bovik, "The mutli-component AM-FM image representation,” IEEE Trans. Image Processing, vol. 5, pp. 10941100, June 1996.

[24] C. Park, J. Lee, M. Smith, and K. Park, "Iris-based personal authentication using a normalized directional energy feature," in Proc. 4th Int. Conf. Audio- and Video-Based Biometric Person Authentication, 2003, pp. 224-232.

[25] B. Kumar, C. Xie, and J. Thornton, "Iris verification using correlation filters," in Proc. 4th Int. Conf. Audio- and Video-Based Biometric Person Authentication, 2003, pp. 697-705.

[26] P. Hong and M. 1. T. Smith, "An octave-band family of nonredundantdirectional filter banks", IEEE proceedings of ICASSP, vol. 2, pp.1165-1168, 2002.

[27] Y. Huang, S. Luo, and E. Chen, "An efficient iris recognition system," in Proceedings of the First International Conference on Machine Learning and Cybernetics, China, November 2002, pp. 450-454.

[28] L. Ma, T. Tan, Y. Wang, and D. Zhang, "Efficient iris recognition by characterizing key local variations," Image Processing, IEEE Transactions on, vol. 13, no. 6, pp. 739-750, June 2004

[29] J. Behar, M. Porat, and Y.Y. Zeevi, "Image Reconstruction from Localized Phase," IEEE Trans. Signal Processing, vol. 40, no. 4, pp. 736-743, Apr. 1992.

[30] GU, X., GORTLER, S., AND HOPPE, H. 2002. Geometry images. ACM SIGGRAPH 2002, pp. 355-361.

[31] SANDER, P,WOOD, Z., GORTLER, S., SNYDER, J., AND HOPPE, H. 2003. Multi-chart geometry images. Eurographics Symposium on Geometry Processing 2003, pp. 146-155.

[32] PRAUN, E., AND HOPPE, H. 2003. Spherical parametrization and remeshing. ACM SIGGRAPH 2003, pp. 340-349.

[33] LOSASSO, F., HOPPE, H., SCHAEFER, S., AND WARREN, J. 2003. Smooth geometry images. Eurographics Symposium on Geometry Processing 2003, pp. 138-145.

[34] BRICEÑO, H., SANDER, P, MCMILLAN, L, GORTLER, S., AND HOPPE, H. 2003. Geometry videos: A new representation for 3D animations. ACM Symposium on Computer Animation 2003, pp. 136146.

[35] M. H. Mahoora and M. Abdel-Mottalebb, "Face recognition based on 3D ridge images obtained from range data," Pattern Recognition, Vol. 42, pp. $445-451,2009$.

[36] K.W. Bowyer, K.Chang, and P. Flynn, "A survey of approaches and challenges in 3D and multi-modal3D $+2 \mathrm{D}$ face recognition," Computer Vision and Image Understanding, Vol. 101, No.1, pp. 1-15, 2006.

[37] A.F. Abate, M. Nappi, D. Riccio and G. Sabatino, "2D and 3D face recognition: A survey," Pattern Recognition Letters, Vol. 28, pp. 18851906, 2007.

[38] K. I. Chang, K. W. Bowyer and P. J. Flynn, "Multiple nose region matching for 3D face recognition under varying facial expression," IEEE Transactions on Pattern Analysis and Machine Intelligence, Vol. 28, pp. 1695-1700, 2006.
[39] G. Günlü and H. S. Bilge, "Face recognition with discriminating 3D DCT coefficients," The Computer Journal, Vol. 53, No. 8, pp. 13241337, 2010.

[40] L. Akarun, B. Gokberk, and A. Salah, "3D face recognition for biometric applications," In Proceedings of the European Signal Processing Conference, Antalaya, 2005.

[41] Ramadan, Rabab M., and Rehab F. Abdel-Kader. "3D Face Compression and Recognition using Spherical Wavelet Parametrization." International Journal of Advanced Computer Science and Applications (IJACSA) 3.9 (2012).

[42] Masek, Libor. Recognition of human iris patterns for biometric identification. Diss. Master's thesis, University of Western Australia, 2003.

[43] Kulkarni, S. B., R. S. Hegadi, and U. P. Kulkarni. "Improvement to libor masek algorithm of template matching method for iris recognition." Proceedings of the International Conference \& Workshop on Emerging Trends in Technology. ACM, 2011.

[44] Kevin W. Boyer, Karan Hollingsworth and Patrick J. Flynn. Image understanding for iris biometric: A survey Computer Vision and Image Understanding 110 (2), 281-307, May 2008.

[45] Richard Yew Fatt Ng, Yong Haur Tay and Kai Ming Mok. An Effective segmentation method for iris recognition system, The Institution of Engineering and Technology, 2008, PP-548 to 553

[46] P. Alliez and C. Gotsman, "Shape compression using spherical geometry images," In Proceedings of the Symp. Multi-resolution in Geometric Modeling, 2003.

[47] Praun and H. Hoppe, "Spherical parameterization and remeshing," in ACM SIGGRAPH 2003, pp. 340-349, 2003.

[48] L. Pastor, A. Rodriguez, J. M. Espadero, and L. Rincon, "3D waveletbased multi-resolution object representation," Pattern Recognition, Vol. 34, pp. 2497-2513, 2001.

[49] M. Alexa, "Recent advances in mesh morphing," Computer Graphics Forum, Vol. 21, No. 2, pp. 173-196, 2002.

[50] X. Gu, S. J. Gortler and H. Hoppe, "Geometry images," ACM SIGGRAPH, pp. 355-361, 2002.

[51] P. Schröeder and W. Sweldens, "Spherical wavelets: Efficiently representing functions on a sphere," In Proceedings of Computer Graphics (SIGGRAPH 95), pp. 161-172, 1995.

[52] P. Schröder and W. Sweldens, "Spherical wavelets: Texture processing," in Rendering Techniques, New York, 1995, Springer Verlag.

[53] P. Schröeder and W. Sweldens, "Spherical wavelets: efficiently representing functions on a sphere," In Proceedings of Computer Graphics (SIGGRAPH 95), pp. 161-172, 1995.

[54] S. Campagna and H.-P. Seidel, Parameterizing meshes with arbitrary topology. In H.Niemann, H.-P. Seidel, and B. Girod, editors, Image and Multidimensional Signal Processing' 98, pp. 287-290, 1998.

[55] P. Yu, P. Ellen Grant, Y. Qi, X. Han, F. Ségonne and Rudolph Pienaar, “ Cortical surface shape analysis based on spherical wavelets," IEEE Transactions on Medical Imaging, Vol. 26, No. 4, pp. 582-597, 2007. AUTHORS PROFILE

Rabab Mostafa Ramadan attended Suez Canal University, Port-Said, Egypt majoring in Computer Engineering, earning the BS degree in 1993. She graduated from Suez Canal University, Port-Said, Egypt with a MS degree in Electrical Engineering in 1999. She joined the Ph.D. program at Suez Canal University, Port-Said, Egypt and earned her Doctor of Philosophy degree in 2004. She worked as an Instructor in the Department of Electric Engineering (Computer division), Faculty of Engineering, Suez Canal University. From 1994 up to1999, as a lecturer in Department of Electric Engineering (Computer division), Faculty of Engineering, Suez Canal University From 1999 up to 2004, and as an assistant Professor in Department of Electric Engineering(Computer division), Faculty of Engineering , Suez Canal University From 2004 up to 2009. She is currently an Assistant professor in Department of Computer Science, College of Computer \& Information Technology, Tabuk University, Tabuk, KSA. Her current research interests include Image Processing, Artificial Intelligence, and Computer Vision. 